\title{
PHISHING, TRUST AND HUMAN WELLBEING
}

\author{
John Ayooluwa Adejobi ${ }^{1}$, Fiona Carroll ${ }^{1}$ and Liqaa Nawaf ${ }^{1}$ and Reza Montasari ${ }^{2}$ \\ ${ }^{1}$ Cardiff Metropolitan School of Technologies \\ Cardiff Metropolitan University, Llandaff Campus, Western Avenue, Cardiff, CF5 2YB, Wales, UK \\ ${ }^{2}$ Hillary Rodham Clinton School of Law, Swansea University \\ Swansea University, Singleton Park, Swansea, SA2 8PP, Wales, UK
}

\begin{abstract}
The more time we spend online, the more our global connections grow and frighteningly, the more we are susceptible to cyberattacks. In particular, 2020/ 2021 has seen a significant increase in successful phishing attacks mainly due to the rise in human vulnerabilities caused by the global COVID-19 situation. In this paper, we consider the human factors of the phishing attack. We explore the evolution of the phishing attack with particular reference to its many different guises. Following that, the paper discusses the concept of trust and the human trust issues resulting from a phishing attack; it will focus on human vulnerability and the idea of phishing awareness. The findings from the study show that people feel that they can confidently recognize a phishing attack but it also highlights how personal attitudes and situational influences can make certain online users more susceptible than others. The findings also clearly detail how the phishing attack makes them and others feel and they assertively suggest ways to counter the attack. After portraying this picture, the question lies around the degree to which the everyday person is adequately equipped to psychologically deal with the sophisticated and contemporary phishing attack, and whether their thinking and methods of detection/ coping are so outdated that this (in itself) is creating an even greater risk.
\end{abstract}

\section{KEYWORDS}

Phishing, Trust, Wellbeing, Cyber Security, Vulnerability

\section{INTRODUCTION}

The COVID-19 pandemic has brought with it an avalanche of cyber-attacks and in particular phishing attacks. Cyber criminals are targeting people's vulnerabilities caused by the uncertainty of the virus as well as their increased dependence on remote working and digital tools. This last year, the UK Department for Digital, Culture, Media \& Sport (2020) has experienced a rise in businesses experiencing phishing attacks (from $72 \%$ to $86 \%$ ) (DCMS, 2020). Indeed, whether corporate or personal, the phishing attack is on the rise and many people, all over the world, are battling to detect and cope with these threats and attacks. Despite the fact that the phishing has been around a long time (i.e. as long as email has existed), it continues to present a substantial risk to people and their wellbeing. The question arises: why are we still so susceptible and vulnerable to the phishing attack? In this paper, we take a glimpse into the everyday online user's understanding and experience of the phishing attack. We will focus on trust and human trust issues resulting from a phishing attack and in doing so, we will build a more human centric picture of where the problem/s lie. This paper is organized as follows. Section 2 discusses the evolution of the phishing attack focusing on the changing anatomy of the phishing attack. Section 3 discusses trust, vulnerability and human wellbeing. Section 4 documents the study and its main findings. Finally, section 5 reviews future investigations and concludes the paper.

\section{THE CHANGING ANATOMY OF THE PHISHING ATTACK}

Phishing attacks evolved with the introduction of various technology. For example, the introduction of mobile phones and application software have made users much more vulnerable to phishing attacks, giving criminals/ phishers more opportunities for exploitation. In particular, a major part of application software 
being exploited by phishers is the email. 'In terms of scope, physical infrastructure, usage, and accessibility, email is of killer application proportions'(Tassabehji \& Vakola, 2005, p.70). The phisher's objective, generally, is to lure their victim to another interface to gain sensitive information and often, to obtain financial data. In fact, the phishing email is a 'subset of spam which is related to social engineering schemes, which depends on forged e-mails (i.e. it claims that originated from a legitimate company or bank) and then through an embedded link within the e-mail, the phisher tries to redirect users to fake websites' (Almomani et al., 2013, p.1). Concisely, phishing attacks have evolved from 1) the email with wrong spellings and grammar to 2) the email presenting a desperate urgency to click a link to 3) the email with professional and very sophisticated ways of obtaining data. And social media has a huge impact on the success of these more sophisticated attacks. For example, social media has made spear phishing much easier. Spear phishing is a more targeted version of phishing "where an adversary conducts online reconnaissance against an individual or organization in order to construct an email which appears to be of significant interest to those targeted' (CPNI, 2021, p.1). The email is strategically designed to persuade the person to open a file attachment or click on a website link. Indeed, the art is in the persuasion and as we have seen particularly during COVID-19, phishers have been evolving their methods and techniques to persuade, they capitalise on human vulnerabilities and manipulate people's trust to achieve their malicious objectives.

Some of the earliest techniques (human and technical) that have been used to detect phishing attacks have centred on the presentation and appearance of the emails. These include things like: misspelled URLs, grammatical errors, hidden URLs, etc. However, this becomes much more difficult today as phishers have improved on these basic inaccuracies and mistakes, it is now a lot harder for a human and machine to detect a syntax pattern used by phishers. Indeed, using semiotic mechanisms, phishing attackers can make victims believe that they are communicating with a trusted entity or brand, this is often executed through websites displaying official logos to obtain sensitive information. In fact, a visual deception can make many technical securities redundant as the onus is on the users to identify contents of such signs and symbols being used. As Dhamija (2006, p.1) highlight 'some visual deception attacks can fool even the most sophisticated users. These results illustrate that standard security indicators are not effective for a substantial fraction of users and suggest that alternative approaches are needed'.

A phishing attack has been described as a clever array of three forms of pattern: authority, time pressure and tone (Wanca \& Cannon, 2016). Trusted brand logos create and provide the necessary illusion of legitimacy (and authority) to users and they open them up to phishing attacks. As Wanca and Cannon (2016, p.8) show 'Phishing scams claim to be from a trusted source by using a corporate logo or name as the sender to attempt to create legitimacy and credibility'. Time is another important factor used to lure victims into making decisions without thinking it through. Time can put them under a lot of pressure especially when needing to make a decision on whether to trust or not trust. Finally, the tone in which the message is delivered is another crucial component. For example, a formal tone with a combination of persuasive and courteous statements has the power to influence and manipulate the user's decision making (Wanca \& Cannon, 2016). In line with this, it is no longer about sending a single isolated phishing email. Social engineering is a complex approach now used by attackers to mislead and manipulate users to ensure a more successful phishing attack. It is less predictable and often more calculated: 'Social engineering is a type of psychological manipulation. The attacker will persuade you to undertake a number of actions or to provide information that may seem innocent to you, by pretending to be someone else' (BDO, p.4). There are many types of social engineering approaches that can all link up to ensure a more convincing attack and in terms of phishing, these are no longer surface 'syntax' deep. They are now a deeper and more studied psychological attack which probes and persuades the human in clever ways.

\section{TRUST AND HUMAN WELLBEING}

Trust is a key player in the phishing attack. The question remains, why, despite receiving wide media coverage, has the globally discussed and known phenomenon of the phishing attack continued to be effective? For example, during COVID-19, the preying on people's vulnerabilities and the securing of people's trust was/is the main trigger for a continued successful phishing attack landscape. People tend to believe/ accept familiarity, as discussed, they trust professional looking brands and emails. However, it goes without saying that 'email has always been a casual communications medium: email content is not 
authenticated, and forging an email is an easy operation' (Adida et al.,2005, p. 2). By researching a person presence online (i.e. life), phishers can gather not only people's history but also their current interests, hobbies, beliefs, likes, dislikes etc. A lot of valuable data can easily be obtained from online platforms; in fact, sufficient information can be collected to fool them into thinking that they are in contact with a familiar person. Like a spider, this information can help attackers to fabricate environments in the form of convincing interfaces and links to lure victims into giving out sensitive information. 'Human intellectual and social life is based on the production, use, and exchange of signs. When we gesture, talk, write, read, watch a TV program, listen to music, navigate through YouTube, or look at a painting, we are engaged in sign-directed or meaning-based behavior' (Danesi, 2019, p.37). The phishing experience can be built from these signs and can seem very authentic. Hence it's detection can be very difficult. 'One of the biggest challenges facing businesses in their fight against cyberthreats is the breadth of phishing scams used' (Rutherford, 2018, p6-8). With a world of private data being freely uploaded on social media, attackers can apply their skills in many different ways to gain sensitive information. 'In today's technologically connected society, social engineers have begun to focus and apply their skills onto the cyber realm in hopes of gaining access to unauthorized or private information' (Walker, 2016). Research has shown that an emphasis on persuasive scams and social engineering are key elements to ensuring phishing attack success (Ferreira \& Lenzini, 2015).

As the UK Department for Culture, Media \& Sport (DCMS, 2020) highlight from 2017 to 2020 there has been a rise in phishing attacks (it increased by around 14 percent). Anti-phishing tools are becoming more and more ineffective when attackers use human behaviors and trust mechanisms to carry out such fraudulent attacks. As Abroshan et al. (2018) show phishers invest a lot of time in the design of phishing techniques solely around human behaviours. With that, there is an interplay of emotion which often impacts users' judgments on phishing attacks; these attacks strategically use enticing and emotive information to lure victims into giving sensitive information. Indeed, emotions have a strong impact on trust decisions: 'It has long been known that the principal determinant of human behavior is emotion, that when the emotions and intellect are in competition for control of will, it is usually that emotion that win' (Arnold, 1970, p31). To the extent, social-psychological research has determined ineffective cognitive processing as a key reason for individual victimization (Harrison et al., 2016). And social media tends to create a culture of false trust which drives relationships and promotes the sharing of excess information. Unfortunately, this façade of false trust and hence humans consciously and often unconsciously sharing their information (i.e. users habits and social lives) is having an impact on human wellbeing and in doing so, also appears to be the root to many phishing susceptibilities. In detail, human wellbeing is a complex ecosystem including emotional and intellectual aspects. Phishing attacks that deceive and take an advantage of human emotions can lead to fear and distrust which in turn leads to isolation and a loss of relationships and lower quality of life (Drevitch, 2021).

\section{STUDY}

The goal of this study was to explore the human factors of the phishing attack experience and to share a glimpse of phishing through the eyes of the everyday online user. The study took place at Cardiff Met University in Autumn 2020 and aims to give insight into individuals' understanding and feelings around the phishing attack and the impact that it has on a person. Ninety-two participants from both the UK and Nigeria completed the study. The study took approximately twenty minutes in duration. The study was approved by the Ethics Board of School of Technologies, Cardiff Met University and subjects provided online consent for study participation and the academic use of de-identified data.

\subsection{Data Collection}

The study was conducted using the Qualtrics online survey software. Participants were presented with a series of quantitative and qualitative questions. To detail, some of these questions included: In your opinion, how would you spot a phishing attack? Do you think phishing attacks has changed since you have been online? Why do you think you were a victim to phishing attack? The study also collected data on participants descriptions of the phishing attack: How would you describe a phishing email attack to a friend? Participants were also asked: In general, how did you/ how do you think people feel when they get a phishing email attack? And in your opinion, what is the impact/outcomes of a phishing attack on a person? 


\subsection{Methodology}

A survey was carried out via questionnaires on Qualtrics software to get different perspective to phishing attacks. It examined different ages, educational groups, race, gender and two counties. During the research, samples of the participant were collected which was used to represent the group. Probability sampling was used, making strong statistical inferences about the whole group. Data gathered were analyzed using excel. Social media (twitter) analysis was carried out checking sentiments of key words such as Phishing email, scam, financial loss etc. Participants were presented with a series of quantitative and qualitative questions in other to get their perception to phishing attacks. Data collected were analyzed thus, bringing several findings to the survey.

\subsection{Findings}

After an analysis of the collected data, several interesting findings were identified. For starters, most participants $(82 \%)$ shared that they depend on grammatical errors, spelling mistakes and obvious irregularities in emails to spot phishing attacks. In detail, many of participants felt they could spot such attacks:

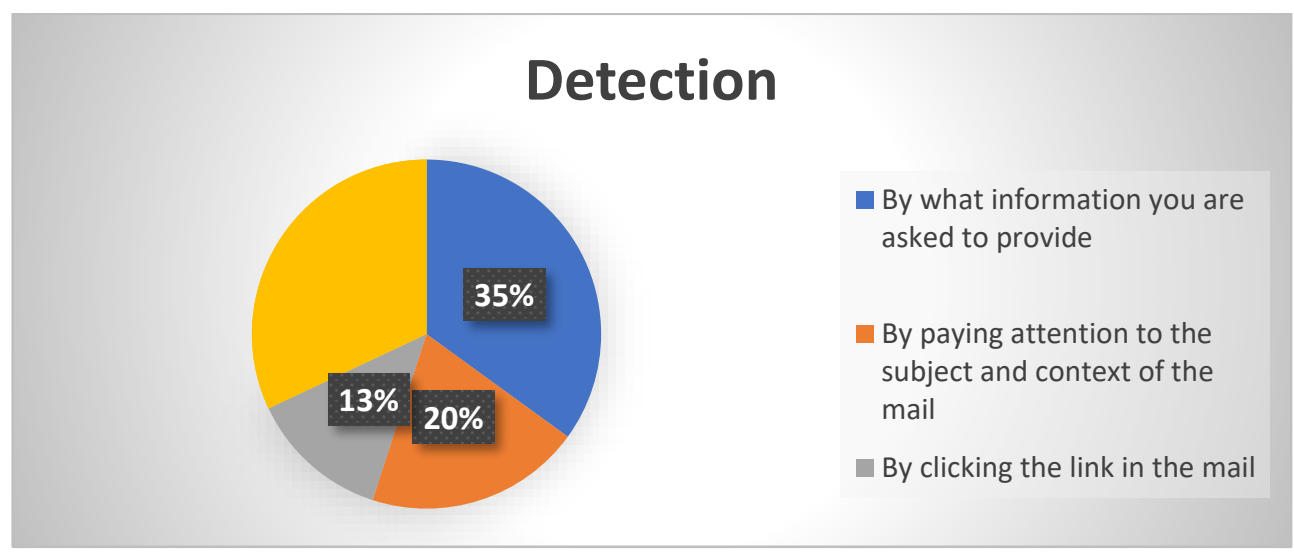

Figure 1. Detection of the phishing email attack

By what information you are asked to provide (35\% participants)

- $\quad$ Asking for personal information (participant 54)

- Through the info being sent to me and what I am being asked to provide(participant 79)

- Usually when they request for personal information such as bank details claiming to be a representative of my bank (participant 80 )

- $\quad$ They tend to want immediate response with threats (participant 87)

- Combination of things such as grammar, the address it was sent from, the content on the email and what it is asking for, whether I was addressed by name and also whether I was expecting that particular email (participant 31)

By paying attention to the subject and context of the mail (20\% participants)

- $\quad$ Spelling mistakes- Dodgy email accounts - non-relevant information (participant 19)

- Formatting, grammar, but also the context and reasoning given for personal data requests (participant 22)

- Incorrect sender address, missing details like starting the email with "Dear user" instead of the person's name. Spelling errors or missing data from the email or website. (participant 40)

- Negative perceptions, unnecessary distractions and fake news (participant 58)

- Their emails are usually not encrypted (i.e. not secure), appear in your spam box and websites that lack a secure connection (participant 72)

- There's always a sort of bait, sometimes it's obviously too good to be true (participant 77)

By clicking the link in the mail (13\% participants). 
- spelling errors, looking behind the email picture / name, looking - without clicking - as to where the link points to (participant 20)

- Links asking for personal information (participant 55)

- The link (participant 56)

- Email or web link from unknown source (participant 83)

When participants were asked if they think the phishing attack has changed since they have been online? All participants agree that phishing attacks have evolved from when they first started to go online. The findings show that the majority of participants within the age bracket 17-24 years old (85\%) receive 0-5 malicious emails per week. This compares with the $36 \%$ of the $25-35$ age group, the $20 \%$ of $36-45$ age group and 50\% of 46-55 age group. Interestingly, when participants were asked why do they think they were a victim to phishing attack? The findings shows that around $60 \%$ of participants ignored this question. Around $15 \%$ stated they were victims out of curiosity, negligence, and a weak security. In all responses, it is clear that participants were blaming their own actions for being phished: 'Because I was looking for something online. Or I was trying to get a Job' (participant 57); 'My account was hacked after getting an email' (participant 61); 'My security wasn't strong enough' (participant 78) and 'There was no prize as the link suggested' (participant 84 ).

In more detail, three participants claimed it was as a result of their impatience. As one participant (40) highlighted 'I rushed into things - was foolish and did not take time to check. May have been to tiredness'. Others claimed it was because 'I was in a hurry' (participant 64) and 'I was in a haste' (participant 76). The study also highlighted participant's descriptions of the phishing attack (see Figure 1). Words like 'loss', 'financial loss', 'trust issues', 'attack', 'money', 'account' and 'bad spelling' are featuring most frequently in their descriptions of the phishing attacks.

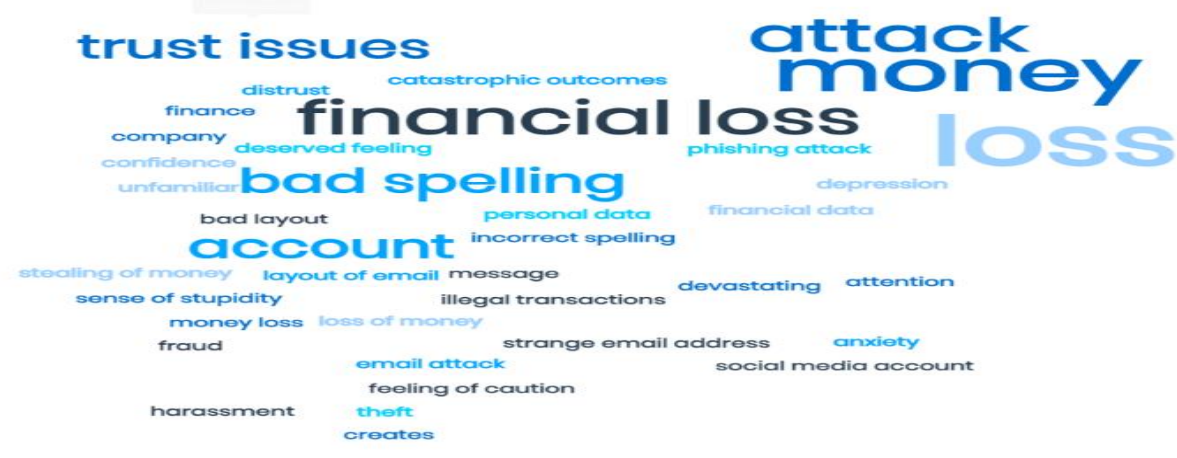

Figure 2. Descriptions of the phishing email attack

Furthermore, a large number of participants discussed the deception in the emails particularly in how the attackers tried to obtain their personal information. Participants were strongly feeling that they were "Fake emails to trick you for information' (participant 12); 'Mass email sent out appearing legitimate trying to bait individuals into a scam or downloading malicious software' (participant 13); 'legit looking email that intends to gain access to sensitive information' (participant 14); 'Looks like it comes from a legit business, will ask for personal info or ask you to click a link within the email or download an attachment' (participant 18); 'An email that copies as much as it can about a real trustworthy company, but it steals your information when you submit it' (participant 35) and finally, 'When the content of the mail includes a link that directs you to a different webpage. Or when a link pops up on your browser. It could also be an SMS telling you to confirm login details and the likes' (participant 47). When participants were probed about how they feel/ think people feel when they get a phishing email attack? Participants aged thirty-five years old and below who answered, believed that people feel nervous, insecure, unaware and less confident after receiving a phishing attack email. Interestingly, the data shows that only two participants aged thirty-six years old and above $1.94 \%$ of overall sample) answered the question. Are these questions too close for comfort? Is there a disconcerted feeling in being fooled (i.e. losing control of one's emotions and trust in people?). Moreover, all the participants from Nigeria (36 participants in total) didn't answer this question. This raises another interesting question: are different cultures more sensitive (and possibly more vulnerable) to phishing attack emails than others? 


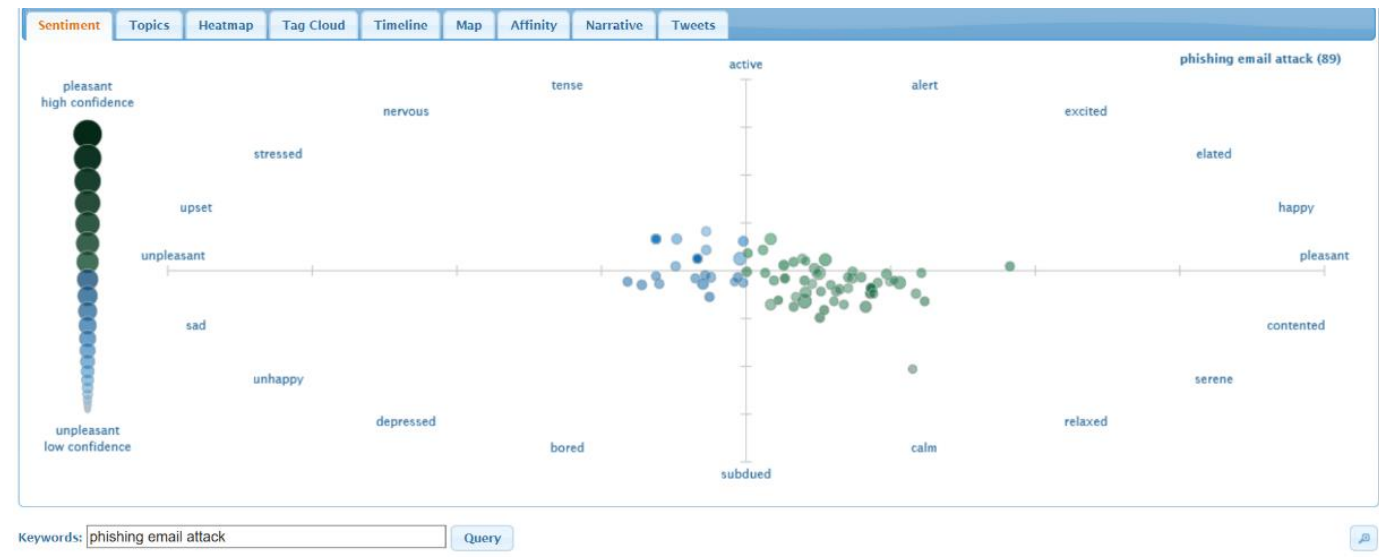

Figure 3. Twitter sentiment

An additional short analysis was carried out on social media data (2nd of February 2021, from 11:00 to 12 midnight) to more generally probe people's feelings to the phishing email attacks. Using the key words "phishing email attacks", the findings highlight that the overall sentiment across all the tweeters was quite negative with words like 'malicious', 'obfuscation', 'warning', 'targeted', 'ransomware' and 'scam' appearing most frequently. It is clear from this data that phishing email attacks afford negativity, the challenge is that even for the more cautious users it is difficult to detect the threat. In many ways, they are alone in this digital threat landscape. Moreover, when participants were asked how confident they were that the government/banks would compensate them for losses due to phishing attack emails. The findings show that all participants from Nigeria seemed to show little trust or confidence in getting a compensation from their government/ banks (i.e. they selected options of 'probably not', 'definitely not' and 'might or might not'). In contrast, participants from the UK felt a bit more confident in getting a compensation from their government/banks (i.e. selecting 'probably yes', 'definitely yes' and a few 'might or might not').

\subsection{Discussion}

From this study, some interesting questions emerge: why are people still susceptible to phishing attacks? And how is this lack of power and lack of knowing what is a threat or not, having on people's wellbeing? The paper shows that a lot of people receive and are still victims to phishing attacks. Indeed, the increase of public awareness on phishing email attacks has reduced the human's contribution to the successful phishing attack campaigns.

However, as the study highlights, even with awareness of these threats, people do still fall to the more sophisticated scams; this is mainly the result of their natural human characteristics such as curiosity, emotions, impatience, laziness and others. As humans, we just can't help ourselves and we fall victim to the psychological tricks, we succumb to the temptation despite often feeling that it could be an inherent risk. Indeed, the impact or outcome of this on a person's wellbeing can be catastrophic. In the study, participants believed that people can feel a lot of stress as a result of a phishing attack, that they can develop anxiety, or have long term trust issues afterwards. As one participant (22) mentioned 'it can make them feel less secure online, and less likely to trust what comes into their inbox'. Another participant (24) highlighted that there might be 'a sense of stupidity/ blaming oneself for falling for it. Afterwards, a well-deserved feeling of caution and distrust'. It could be said that people from countries with little support from the government after a successful phishing email scam, tend to be more alert and vigilant, however, this still does not guarantee that they will not become victims.

The psychological based methodologies used to persuade, coerce, or manipulate can be very convincing. As discussed, the phishing email attack has evolved and is now presenting in various sophisticated forms (combining affective, behavioral and cognitive processes) and thus making it more and more difficult for the human alone to detect. When we asked participants about what they think is needed for them to avoid or to detect a phishing email attack. The findings clearly highlighted that participants felt they and others needed a support such as an alert system and/ or pop-up warnings. They suggested several forms of possible alerts: 'possibly an IP address scanner' (participant 16); 'A security notification of some sort? Detailing the dangers 
and things to look out for?' (participant 12); 'traffic light system?' (participant 22) and 'I don't think a general user interface would be sufficient enough to warn a person about a phishing attack (this by itself could be used to inform a user to look out for phishing attacks). However, the implementation of a UI and algorithm (maybe antivirus) could be utilized to warn a user about a phishing attack by including a small alert section above the email/text to inform the user that the email is suspicious. Thus, allow users the option to listen to the alerts or to ignore them (if it is a false positive)' (participant 30).

In terms of detecting phishing email attacks, we have definitely moved on from using 'misspelling and bad grammar' to identify a possible attack, we now need to look at the pragmatics of the email (i.e. the meanings behind the emails and most importantly the context). This study has highlighted that people want and need support to protect themselves from the phishing attack. It is clear that training, education and awareness are only part of the solution, we need to equip people with the systems that will directly enhance their threat perception. Anti-phishing tools need to take a pragmatic approach to understanding the psychological and sociological factors being played as a form of attack. In adopting this practice, future research will be best placed to design 'alert systems' for not only human online safety but also human wellbeing.

\section{CONCLUSION}

Phishing email attacks which have been prolific since the mid-2000's can no longer solely be detected from their presentation and appearance. The authors of this paper believe that exposing the deeper and often hidden meanings afforded by phishing email attacks is the only way to provide people with a better chance in identifying potential threats. Moreover, they feel that it is only when we start to look at the pragmatics of the email attack that we will truly be in a position to curb and protect against the phishing attack. As the paper highlights, people need support to protect themselves against the phishing attack. The focus here needs to be on identifying and understanding the intent of such emails, rather than just identifying poor spelling and grammar. Despite humans being the biggest risk to organizations, we can't and shouldn't want to take them out of the picture. To ensure a flourishing business we need humans to interact, to communicate and to receive and send emails. Trust and human wellbeing are key players in this mix and to further nurture this trust and wellbeing, we need to adopt an approach that looks at the wider context of these attacks. In fact, more attention needs to be placed on the pragmatics of the attack; people's trust/engagement is one of the main triggers to a successful phishing attacks especially during a trendy event or situation across the world (i.e. Covid-19). Realizing the wider trends and context is crucial to understanding the threat. No doubt, and as we have experienced, the form/medium of communication amongst people may change but the fact remains that people will continue to want to interact and trust one another. This is a good thing, interaction and trust are fundamental to our lives and our businesses, we just need to ensure that people are fully equipped and supported to protect themselves (wellbeing), their businesses, and their trust online.

\section{REFERENCES}

Abroshan, H., Devos, J., Poels, G. and Laermans E. (2018). Phishing attacks root causes. In Lecture Notes in Computer Science (including subseries Lecture Notes in Artificial Intelligence and Lecture Notes in Bioinformatics).

Adida, B., Hohenberge, S. and Rivest, R. L. (2005). Fighting Phishing Attacks: A Lightweight Trust Architecture for Detecting Spoofed Emails. Available from: http://people.csail.mit.edu/rivest/AdidaHohenbergerRivestFightingPhishingAttacks.pdf

Almomani, A., Gupta, B. B. Wan, T.-C., Altaher, A. and Manickam, S. (2013). Phishing Dynamic Evolving Neural Fuzzy Framework for Online Detection "Zero-day" Phishing Email. Technical report.

Arnold, M. B. (1970). Feelings and Emotions: The Loyola Symposium. Google Books.

BDO (2019). A Pragmatic Approach to Enhancing Cyber Security. Available from: https://www.bdo.ky/engb/insights/insights-2016-2019/a-pragmatic-approach-to-enhancing-cyber-security

CPNI (National Centre for the Protection of National Infrastructure) (2021). Spear phishing - Understanding the threat. Technical report. Available from: www.cpni.gov.uk/advice/cyber/online-reconnaissance

Danesi, M. (2019). Understanding Media Semiotics. Google Books. 
DCMS (Department for Culture \& Media \& Sport) (2020). Cyber Security Breaches Survey 2020. Technical report.

Dhamija, R., Tygar, J. D. and Hearst, M. (2006). Why phishing works. In Conference on Human Factors in Computing Systems - Proceedings. DOI 10.1145/1124772.1124861

Drevitch, G. (2021). How Does Fraud Impact Emotional Well-Being? Available from: https://www.psychologytoday.com/us/blog/the-fraud-crisis/202101/how-does-fraud-impact-emotional-well-being

Ferreira, A. and Lenzini, G. (2015). An Analysis of Social Engineering Principles in Effective Phishing. In Proceedings 5th Workshop on Socio-Technical Aspects in Security and Trust, STAST 2015.

Harrison, B., Svetieva, E. and Vishwanath, A. (2016). Individual processing of phishing emails: How attention and elaboration protect against phishing. Online Information Review.

Rutherford, R. (2018). The changing face of phishing. Computer Fraud and Security.

Tassabehji,R. and Vakola, M. (2005). Business email: The killer impact. Commun. ACM. 48. 64-70. 10.1145/1096000.1096006.

Walker, L. E. (2016). Deception of Phishing: Studying the Techniques of Social Engineering by Analyzing Modern-day Phishing Attacks on Universities. Available from https://etd.auburn.edu/bitstream/ handle/10415/5175/LEW\%20Thesis.pdf?sequence=2\&isAllowed=y

Wanca, I. and Cannon, A. (2016) How human behavior and decision-making expose users to phishing attacks. Technical report. Available from: http://www.nycrimecommission.org/pdfs/CCC-How-Human-Behavior-and-DecisionMaking-Expose-Users-to-Phishing-Attacks.pdf 